\title{
Build Orientation Optimization Problem in Additive Manufacturing
}

\author{
Ana Maria A. C. Rocha ${ }^{1(\bowtie)}\left(\mathbb{D}\right.$, Ana I. Pereira ${ }^{2}\left(\mathbb{C}\right.$, and A. Ismael F. Vaz ${ }^{1}(\mathbb{0}$ \\ 1 Algoritmi Research Centre, University of Minho, 4710-057 Braga, Portugal \\ \{arocha, aivaz\}@dps. uminho.pt \\ 2 Research Centre in Digitalization and Intelligent Robotics (CeDRI), \\ Instituto Politécnico de Bragança, 5300-253 Bragança, Portugal \\ apereira@ipb.pt
}

\begin{abstract}
Additive manufacturing (AM) is an emerging type of production technology to create three-dimensional objects layer-by-layer directly from a $3 \mathrm{D}$ CAD model. AM is being extensively used by engineers and designers.

Build orientation is a critical issue in AM since it is associated with the object accuracy, the number of supports required and the processing time to produce the object. Finding the best build orientation in the AM will reduced significantly the building costs and will improve the object accuracy.

This paper presents an optimization approach to solve the part build orientation problem considering the staircase effect, support area characteristics and the build time. Two global optimization methods, the Electromagnetism-like and the Stretched Simulated Annealing algorithms, are used to study the optimal orientation of four models.

Preliminary experiments show that both optimization methods can effectively solve the build orientation problem in AM, finding several global solutions.
\end{abstract}

Keywords: Design tools $\cdot$ Additive manufacturing $\cdot 3 \mathrm{D}$ printing Optimization · Build orientation

\section{Introduction}

Traditional manufacturing methods involve a solid block of material being carved, or shaped, into the desired product, where block parts are being successively removed in different ways.

Additive manufacturing (AM) has emerged in the last decades becoming an alternative to the traditional subtractive manufacturing. It is a technology that builds $3 \mathrm{D}$ objects by adding ultrathin layers of material, one by one, for fabricating the desired product.

Additive manufacturing processes involve the use of three-dimensional (3D) computer-aided design (CAD) data to create physical models. One of the greatest 
benefits of AM is the production of a wide range of shapes. Currently, AM is being used to make end-use products in aircraft, dental restorations, medical implants, automobiles, and even fashion products [7].

One of the current challenges faced by manufacturing industries is the reduction of prototype model development time through adoption of rapid prototyping technologies (techniques of fabricating a prototype model from a CAD file) in particular using the additive manufacturing process.

The performance of a rapid prototyping technology depends on the way parts are oriented on the build platform. Therefore, each part should be appropriately oriented to achieve better surface quality and either minimal support structure or lower build time [3]. Besides that, these strategies result in a process more environmental friendly since it will require less energy consumption and material waste.

A number of studies have been carried out in the problem of selecting a building direction given a 3D CAD model. A proposal based on the determination of the optimal part orientation when minimizing the build cost can be seen in [1]. Authors used various criteria like stair step error, build height, volume of supports, stability of object, among others, to determine the optimal part build orientation for any rapid prototyping process. Lan et al. [8] studied the best build orientation of a model part considering the surface quality, build time and the complexity of the support structure. Thrimurthulu et al. and Canellidis et al. [3,14] show methodologies addressing the optimal part orientation taking into account the surface quality, evaluate the surface roughness and build deposition time.

A presentation of methodologies for optimizing the build orientation problem based on the minimum volumetric error can be found in [9]. A part orientation optimization model using genetic algorithm that considers the build time, material usage, surface finish, interior geometry, strength characteristics and related parameters is presented in [15].

The literature suggests criteria to be considered for optimal build orientation such as the build height, staircase effect, volume of support structures and part area in contact with support structures $[4,5,12,15]$.

In this study, we aim to analyse the behaviour of two multi-global optimization methods, named Electromagnetism-Like and Stretched Simulated Annealing algorithms, to solve the build orientation optimization problem in additive manufacturing.

This paper is organized as follows. Section 2 presents the build orientation optimization model as well as the mathematical formulation of the optimization problem. In Sect. 3, the optimizers used to solve the build orientation optimization problem are briefly described. The description of the models that will be analysed are presented in Sect. 4 . Section 5 presents the results of the numerical experiments and Sect. 6 contains the conclusions of the present study and future work. 


\section{Build Orientation Optimization}

In this section the build orientation model as well as the mathematical formulation of the build orientation optimization problem are presented.

\subsection{Build Orientation Model}

The surface finish of an object obtained through additive manufacturing process is highly important. Different measures to determine the best build orientation for an improvement of the surface finish can be considered taking into account factors as the part accuracy, building time, structure support and part stability. The general orientation characteristics for assessing the optimal build orientation include: the height of the part in the build direction; the total volume of support material used; the total area of contact of the object with the external support structure; the quality of selected faces that are subjected to the staircase effect and are in contact with the supports. These orientation characteristics can be individually used to rank a selection of possible orientations according to minimum build height, minimum volume of support structures, minimum area in contact with extra support structures or maximum accuracy of total surface area (staircase effect) or selected facets $[5,8,15]$. The best selection of the build orientation model will improve the surface accuracy of the object, minimize the supports needed and the production time, and, consequently, the build costs [1].

A major source of structure inaccuracy is due to the staircase effect. The staircase effect is used as the basis for developing an accuracy measure. The maximum deviation from layered part to the CAD surface measured in the normal direction to CAD surface is known as the cusp height, $H_{c}$ (see Fig. 1).

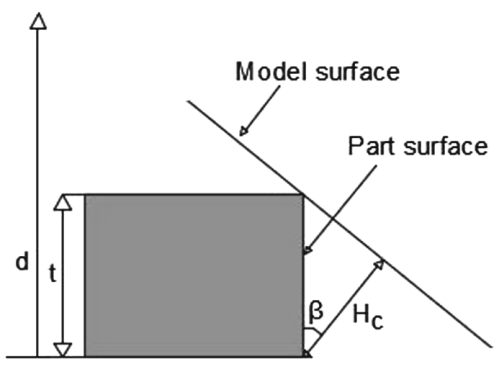

Fig. 1. Cusp height.

This depends on the angle $\beta$ formed by the slicing direction $d$ and the model surface normal, and on the layer thickness, $t$. Thicker layers and/or higher values of $\cos (\beta)$ will produce larger values for cusp height and consequently a more inaccurate surface will appear [1]. The cusp height is given by $H_{c}=t \cos (\beta)$. By using cusp height to measure the structure accuracy, the surface quality can be determined from the object geometry, build direction and layer thickness. 
When the normal model surface is close to be collinear $(\beta \approx 0)$ with the slicing direction, the volumetric difference (difference between CAD model and the slice volume) is very high, giving a low surface smoothness.

However if the normal of the model surface is close to be perpendicular to the slicing direction $\left(\beta \approx 90^{\circ}\right)$ the volumetric difference is very low, giving a high surface smoothness. In this context, an algorithm that slices the model along the slicing direction $d$ can use the volumetric error to maximize the part smoothness. The volumetric error, $V E$, to be minimized in each layer stem from staircase effect is given by

$$
V E=\sum_{j} \frac{t^{2}\left|d \cdot n_{j}\right| A_{j}}{2},
$$

where $t$ is the slicing height, $n_{j}$ is the unit normal vector of the $j$ triangle facet, $A_{j}$ is its area, $d$ is the unit normal vector of the building direction [16].

Another measure related to the optimal build orientation is the quantity of supports used, measured by the support area or support volume. The computation of the support volume is very complex. If the shape of the object is convex, the support volume is the volume of the region lying between the object solid of the part and the platform, the vertical polyhedral cylinder which is bounded below by the platform and above by the object facets whose outward normal point downward. But if the product shape is nonconvex, the problem is more complex, since the supports for some faces may actually be attached to other facets instead of to the platform [16]. The quantity of support area affects post-processing and surface finish. Thus, support area is the total area of the downward-facing facets. Note that the support area have more significant impact on the object accuracy than the support volume. So the part building direction optimization accounts for the support area [16]. The support area, $S A$, to be minimized is expressed as follows

$$
S A=\sum_{j} A_{j}\left|d \cdot n_{j}\right| \delta
$$

where $\delta$, a threshold function, is given by

$$
\delta=\left\{\begin{array}{l}
1 \text { if } d \cdot n_{j}<0 \\
0 \text { if } d \cdot n_{j}>0
\end{array}\right.
$$

Another measure that could be used to determine the optimal part build orientation is the build time without compromising the surface quality.

Canellidis et al. [3] considered that the build time includes the time required to manufacture the object as well as the time required for support removal and surface finishing. The major structure of the overall build time is the creation time of a designed object, whereas the time required for removal of supports and surface finishing is only a minor fraction of build time. It should be noted that different authors considered different definitions for the build time. For example, Lan et al. [8] only considered the build time as the time for creating a designed object 
while Zhao [16] considered the build time as the scanning time and the preparation time. The scanning time includes solid scanning time, contour scanning time and support scanning time, where the solid and contour scanning times are independent of the part building direction and the support scanning time depends on the volume of supports. The preparation time includes the time required to move down the platform during the re-coating, the scraping time and other preparation times. Moreover, the preparation time is dependent on the total number of layers, while the number of layers is dependent on the height of part building direction. Therefore, minimizing the height of the part building direction and, consequently, the total number of layers can reduce the part building time. The part building time, $B T$, to be minimized can be expressed as follows

$$
B T=\max \left(d \cdot v_{1}, d \cdot v_{2}, \ldots, d \cdot v_{n}\right)-\min \left(d \cdot v_{1}, d \cdot v_{2}, \ldots, d \cdot v_{n}\right)
$$

where $v_{i}$ are the vertex triangle facets.

\section{$2.2 \quad$ Problem Formulation}

Based on the part build orientation, the optimization problem will minimize the volumetric error, supporting area and build time defined in Eqs. (1)-(3). We aim to compute the optimal slicing direction $d$, which is a normalized vector (i.e. $\|d\|=1$ ). An equivalent mathematical formulation is to compute the rotation along the $x$ and $y$ axis. In our case we considered $d=(0,0,1)^{T}$ as the slicing direction after a rotation along $(x, y)$ angles, where each angle is between $0^{\circ}$ and $180^{\circ}$. Thus, the optimization problem is given by

$$
\begin{array}{ll}
\min & f(x, y) \\
\text { s.t. } & 0 \leq x \leq 180 \\
& 0 \leq y \leq 180
\end{array}
$$

where the objective function to be minimized, $f(x, y)$, is given by (1) for the volumetric error, (2) for the support area and (3) for the part building time.

\section{Optimizers}

In this section, two global optimization methods used to solve the build orientation problem are presented. First, the Electromagnetism-like algorithm is briefly described followed by a description of the Stretched Simulated Annealing algorithm.

\subsection{Electromagnetism-Like Algorithm}

The Electromagnetism-like (EM) algorithm, developed by Birbil and Fang [2], is a population-based stochastic search method for bound constrained global optimization problems that mimics the behaviour of electrically charged particles. 
The method uses an attraction-repulsion mechanism to move a population of points towards optimality.

The EM algorithm simulates the electromagnetism theory of physics by considering each point in the population as an electrical charge that is released to the space. The charge of each point is related to the objective function value and determines the magnitude of attraction of the point over the others in the population. The better the objective function value, the higher the magnitude of attraction. The charges are used to find a direction for the movement of each point. The regions that have higher attraction will signal other points to move towards them. In addition, a repulsion mechanism is also introduced to explore new regions for even better solutions [2].

The EM algorithm comprises four main procedures: "Initialization", "Compute Force", "Move Points" and "Local Search". The main steps of the EM algorithm are presented in the Algorithm 1.

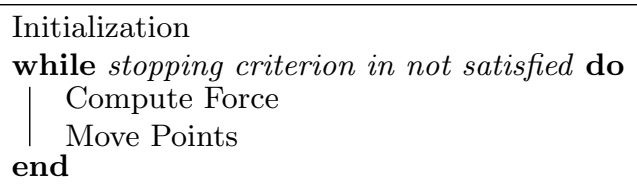

Algorithm 1. EM algorithm

The "Initialization" procedure starts by randomly generating a sample of points. Each point is uniformly distributed between the lower and upper bounds. Then, the objective function value for each point is calculated and the best point of the population, $x^{\text {best }}$, is identified as well as its corresponding objective function value $f^{\text {best }}$.

In the "Compute Force" procedure, each particle charge that determines the power of attraction or repulsion for each point is calculated. In this way the points that have better objective function values possess higher charges. After the charge calculation, the total force vector on each point is then calculated by adding the individual component forces between any pair of points.

The "Move Points" procedure uses the total force vector to move each point in the direction of the force by a random step length $\lambda$. The best point, $x^{\text {best }}$, is not moved. To maintain feasibility, the force exerted on each point is normalized and scaled by the allowed range of movement towards the lower bound or the upper bound.

A fully description of the EM algorithm can be found in [13] as well as the used code.

\subsection{Stretched Simulated Annealing Algorithm}

Stretched Simulated Annealing (SSA) algorithm is a multilocal programming method that solves bound constrained optimization problems point-to-point. This stochastic method combines Simulated Annealing algorithm [6] with a stretching function technique [10]. 
SSA solves a sequence of global optimization problems in order to compute the global/local solutions of the original optimization problem. In each iteration, a new global optimization problem is generate combining the original objective function and the stretching function technique.

The mathematical formulation of the global optimization problem is as follows:

$$
\min _{a \leq x \leq b} \Phi_{l}(x) \equiv\left\{\begin{array}{l}
\hat{\phi}(x) \text { if } x \in V_{\varepsilon^{j}}\left(x_{j}^{*}\right), j \in\{1, \ldots, N\} \\
f(x) \text { otherwise }
\end{array}\right.
$$

where $V_{\varepsilon^{j}}\left(x_{j}^{*}\right)$ represents the neighborhood of the solution $x_{j}^{*}$ with a ray $\varepsilon^{j}$.

The $\hat{\phi}(x)$ function is defined as

$$
\hat{\phi}(x)=\bar{\phi}(x)+\frac{\delta_{2}\left[\operatorname{sign}\left(f(x)-f\left(x_{j}^{*}\right)\right)+1\right]}{2 \tanh \left(\kappa\left(\bar{\phi}(x)-\bar{\phi}\left(x_{j}^{*}\right)\right)\right.}
$$

and

$$
\bar{\phi}(x)=f(x)+\frac{\delta_{1}}{2}\left\|x-x_{j}^{*}\right\|\left[\operatorname{sign}\left(f(x)-f\left(x_{j}^{*}\right)\right)+1\right]
$$

where $\delta_{1}, \delta_{2}$ and $\kappa$ are positive constants and $N$ is the number of minimizers already detected.

The main steps of the SSA algorithm are presented in the Algorithm 2.

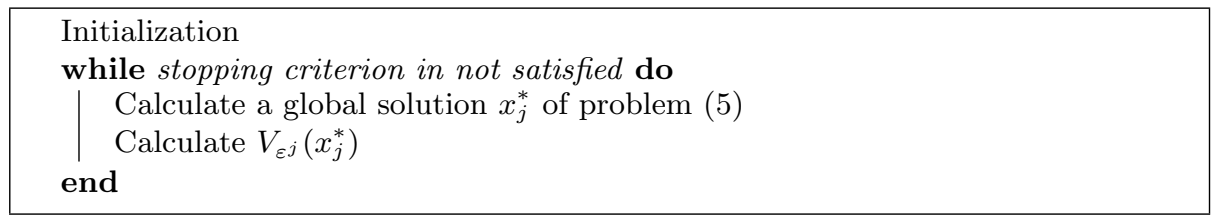

Algorithm 2. SSA algorithm

To solve the global optimization problems (5) the simulated annealing method is used [6]. The SSA algorithm stops when no new optimum is identified after $l$ consecutive runs.

Details about the algorithm and its implementation can be found in [11].

\section{Models Description}

In this section, we present the 3D CAD models that will be used in our numerical testing of the global optimization algorithms.

First, CAD models are converted into STL (STereoLithography) that is the standard file type used by most common 3D printing file formats. STL files describe only the surface geometry of a three-dimensional object without any representation of color, texture or other common CAD model attributes. Figures 2, 3, 4 and 5 show the STL files of the models that will be used in the present study. 


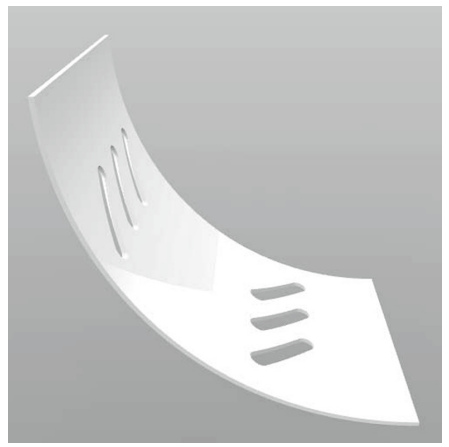

Fig. 2. Rear panel fixed 3D printing file.

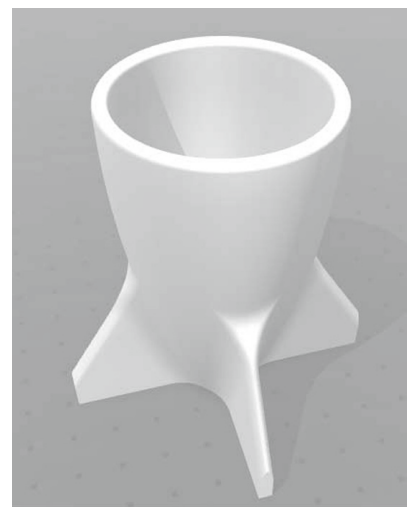

Fig. 4. Rocket shot 3D printing file.

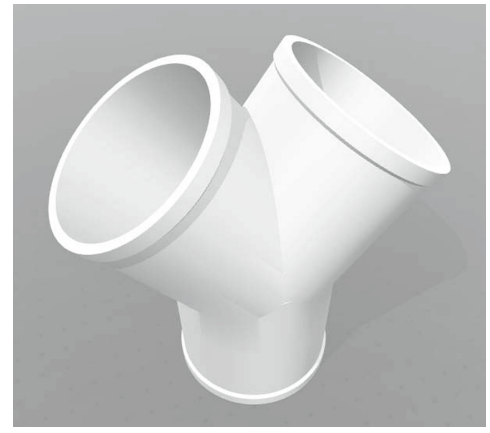

Fig. 3. Air duct 3D printing file.

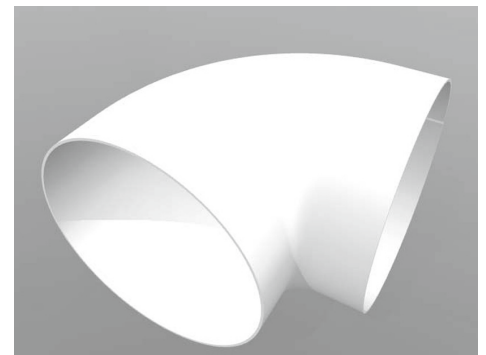

Fig. 5. 45 degree short 3D printing file.

The STL file format approximates the surfaces of a solid model by a polyhedral representation of a 3D object using triangular facets. The more complex the surface is more triangles are produced. Figure 2 shows a rear panel to replace the plexiglass panel on the rear of the Rostock Max v2 (a 3D printer) that has vents on either side. The rear panel is of a difference size of the side panels, but the side panels for left and right are the same. It was defined using 3008 triangles. Figure 3 shows an air duct splitter of $50 \mathrm{~mm}$ designed to direct the flow of two air vents into an air intake or to split the air from one into two places. It was defined using 6024 triangles. Figure 4 shows a rocket shot shaped cup. It was defined using 10616 triangles. Figure 5 shows a fan duct extension with 45 degree short radius elbow that was defined using 66888 triangles.

\section{Numerical Experiments}

In this section, a practical comparison of two optimizers, the EM and SSA described in Sect. 3, to solve the build orientation problem (4) is shown. 
The numerical experiments involve the optimization of the build orientation problem using three different measures: the volumetric error, the support area and the part building time. The models used in the experiments are the ones presented in Sect. 4, namely the Rear panel, Air duct, Rocket shot and 45 degree short.

The numerical experiments were carried out on a PC Intel Core 2 Duo Processor E7500 with $2.9 \mathrm{GHz}$ and $4 \mathrm{~Gb}$ of memory RAM. The algorithm was coded in MATLAB Version 9.2 (R2017a).

\subsection{Details of Implementation}

The stopping criterion used for each optimizer was the maximum number of function evaluations set to 500. Since the presented global methods are stochastic, 20 runs were performed for each problem. The population size used in the EM algorithm was set to 10 .

Before optimization, the objects considered a slicing along the $Z$-axis with $5 \mathrm{~mm}$ height, resulting in 36 slices for the Rear panel fixed problem (see Fig. 6). Considering the same slicing, it was obtained 16 slices for the Rocket shot (see

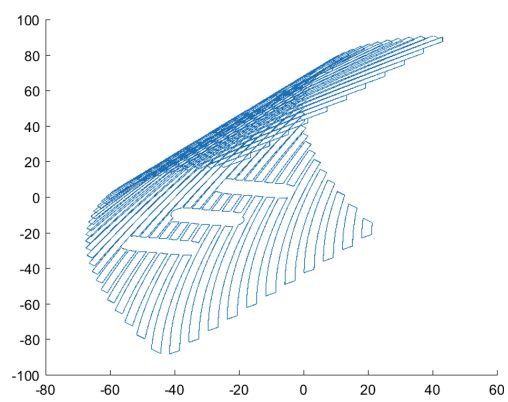

Fig. 6. Rear panel fixed with $5 \mathrm{~mm}$ of layer height.

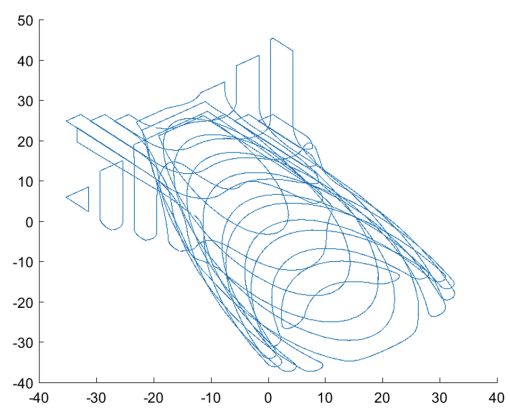

Fig. 8. Rocket shot with $5 \mathrm{~mm}$ of layer height.

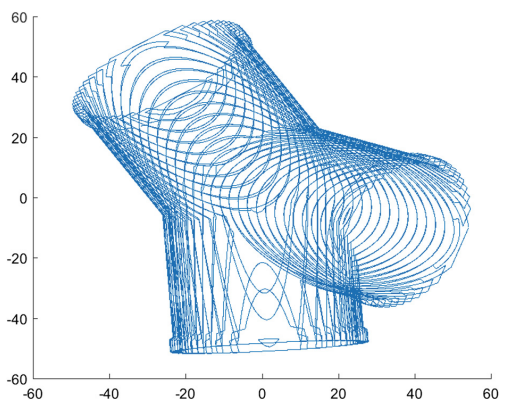

Fig. 7. Air duct with with $2 \mathrm{~mm}$ of layer height.

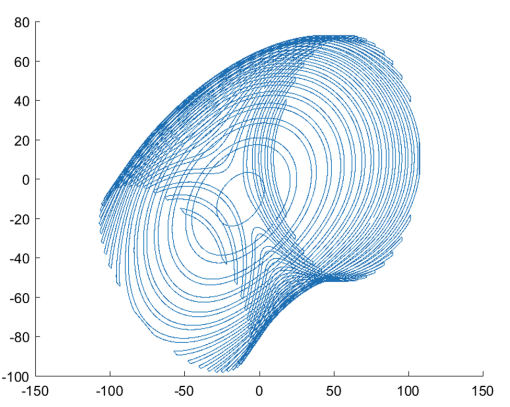

Fig. 9. 45 degree short with $5 \mathrm{~mm}$ of layer height. 
Fig. 8) and 25 slices for the 45 degree short problem (see Fig. 9). For the Air duct problem a slicing along the $Z$-axis with $2 \mathrm{~mm}$ height was used resulting in 49 slices as shown at Fig. 7.

\subsection{Comparative Results}

In this section, we report the numerical results obtained with the Electromagnetism-like and the Stretched Simulated Annealing algorithms.

In the following, we describe the numerical experiences to solve the build orientation problem using the different measures:

- exp_VE - denotes optimizing problem (4) with $f(x, y)=V E$, the volumetric error given by (1);

- $\exp _{-} S A$ - denotes optimizing problem (4) with $f(x, y)=S A$, the support area given by $(2)$;

- exp_BT - denotes optimizing problem (4) with $f(x, y)=B T$, the build time given by (3).

In order to analyse the behaviour of the two global optimization methods in solving the build orientation problem using the different measures, four models were tested: Rear panel fixed, Air duct, Rocket shot and 45 degree short (presented in the Sect.4).

Table 1 presents the numerical solutions, $(x, y)$ in degrees, obtained by the EM and SSA algorithms for each model considered. All different solutions found by the global methods are presented in the Table 1.

The behaviour of the both global optimization methods is very satisfactory since they solve efficiently all the optimization problems, needing few seconds to find the solutions. In general, the solutions found by the methods are very similar and the methods were capable to find more than one solution in each optimization problem.

The EM algorithm found more solutions in two cases (Air Duct \& exp_BT and Rocket Shot \& exp_VE) when compared with SSA algorithm. In some situations both algorithm identified different solutions (45 degree short \& exp_BT).

To evaluate the impact of the different measures to identify the $(x, y)$ on the 3D printer, Figs. 10, 11 and 12 present the build orientation provided by the algorithms when optimizing the build orientation problem of the Rear Panel Fixed model).

The obtained solutions are consistent with the main idea of the measures definition, since in the cases of $\exp _{-} V E$ and $\exp _{-} S A$ the objective is to minimize volumetric error and the support area, respectively.

In the case of the measure $\exp _{-} B T$, where the objective is to minimize the building time, the optimal solution $x=0$ and $y=135$ is consistent with the height of the structure building direction. Note that when the orientation gives the solution of $(180,45)$ the representation of the object is similar to Fig. 12.

Next we will present the figures related to build orientation problem of the Air duct model. Note that the obtained solutions for $\exp _{-} V E$ and $\exp _{-} S A$ are the same, thus the figures of the four solutions are shown in Figs. 13 and 14. 


\begin{tabular}{|c|c|c|c|c|c|c|c|c|c|c|c|c|}
\hline & & $\underbrace{\pi}$ & $\begin{array}{ll}-1 & -1 \\
0 & 0 \\
x & x \\
x & x \\
\dot{+} & \stackrel{+}{+}\end{array}$ & 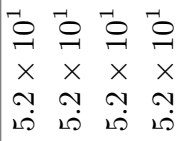 & \begin{tabular}{l}
-1 \\
$\stackrel{1}{1}$ \\
$\times$ \\
\multirow{2}{*}{} \\
in
\end{tabular} & 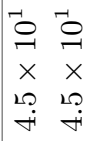 & 11 & 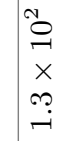 & $\begin{array}{l}\stackrel{\vartheta}{0} \\
\stackrel{-}{\times} \\
\stackrel{\infty}{-}\end{array}$ & 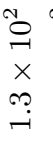 & 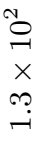 & 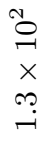 \\
\hline & $\begin{array}{l}\mathbb{S} \\
\tilde{\Omega}\end{array}$ & (8) & 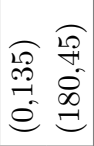 & 尽, & 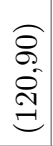 & 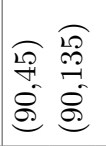 & & $\begin{array}{l}\hat{\theta} \\
\hat{e}\end{array}$ & $\begin{array}{l}\stackrel{2}{\infty} \\
\stackrel{\infty}{=} \\
\infty \\
\stackrel{\infty}{\rightleftharpoons}\end{array}$ & $\begin{array}{l}\stackrel{0}{0} \\
\stackrel{\infty}{\infty} \\
\stackrel{\underbrace{}}{=}\end{array}$ & 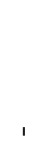 & 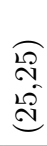 \\
\hline 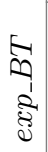 & $\sum_{\text {主 }}$ & .5: & 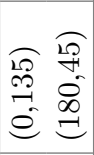 & 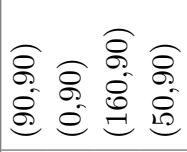 & & 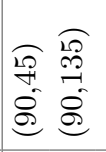 & & $\hat{o}$ & 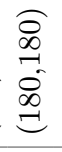 & 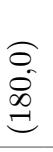 & 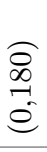 & 1 \\
\hline & & 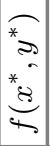 & 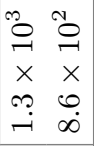 & 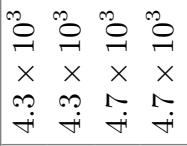 & 1 & 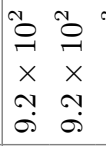 & 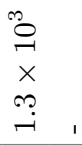 & 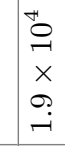 & 1 & 1 & 1 & 1 \\
\hline & $\begin{array}{l}\mathbb{W} \\
\mathbb{2} \\
\mathbb{\Omega}\end{array}$ & 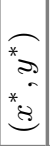 & 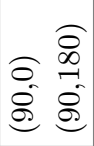 & 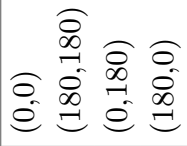 & 1 & 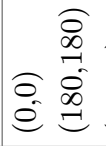 & $\begin{array}{l}\stackrel{0}{0} \\
\stackrel{\infty}{\infty} \\
\stackrel{0}{=},\end{array}$ & 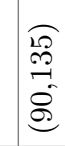 & 1 & 1 & 1 & 1 \\
\hline 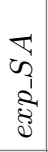 & $\sum_{\text {至 }}$ & 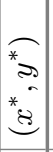 & 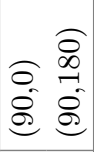 & 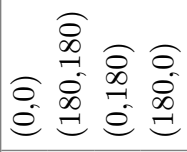 & 1 & 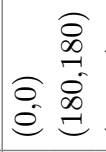 & $\begin{array}{l}\stackrel{0}{0} \\
\stackrel{0}{\infty} \\
\stackrel{D}{=},\end{array}$ & 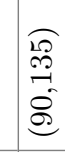 & ' & 1 & 1 & 1 \\
\hline & & 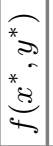 & 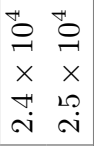 & 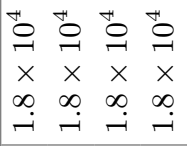 & 1 & 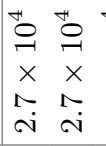 & 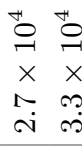 & 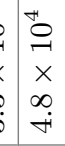 & 1 & 1 & 1 & 1 \\
\hline & $\begin{array}{l}\mathbb{L} \\
\text { 足 }\end{array}$ & 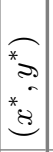 & 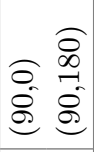 & 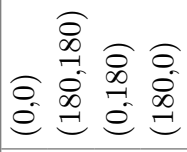 & 1 & $\begin{aligned} & \hat{0} \\
0 & \stackrel{0}{0} \\
\hat{0} & \infty \\
0 & =\end{aligned}$ & & 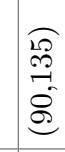 & 1 & 1 & 1 & । \\
\hline 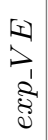 & $\sum_{\text {I }}$ & $\mid$ & 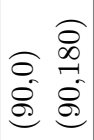 & 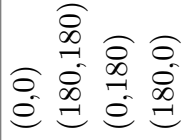 & & 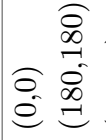 & 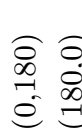 & 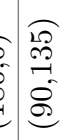 & 1 & 1 & 1 & 1 \\
\hline $\begin{array}{l}\frac{1}{0} \\
\frac{0}{0} \\
0 \\
0 \\
0\end{array}$ & & & 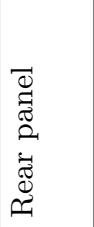 & 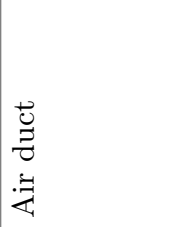 & & 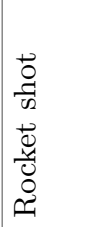 & & 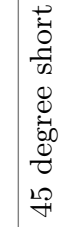 & & & & \\
\hline
\end{tabular}




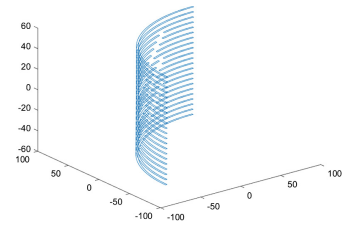

Fig. 10. Rear panel fixed of $\exp _{-} V E$ and exp_SA for $(90,0)$.

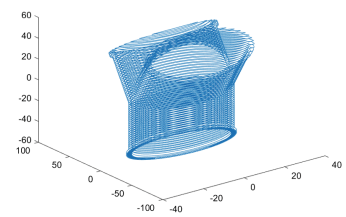

Fig. 13. Air duct of $\exp _{-} V E$ and $\exp _{-} S A$ for $(180,180)$ or $(0,0)$.

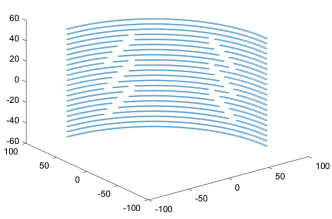

Fig. 11. Rear panel fixed of $\exp _{-} V E$ and exp_SA for $(90,180)$.

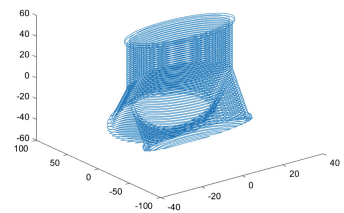

Fig. 14. Air duct of $\exp _{-} V E$ and $\exp _{-} S A$ for $(0,180)$ or $(180,0)$.

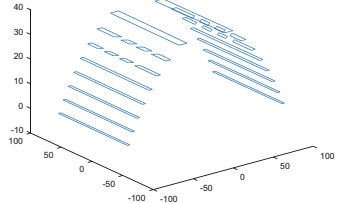

Fig. 12. Rear panel fixed of $\exp _{-} B T(0,135)$.

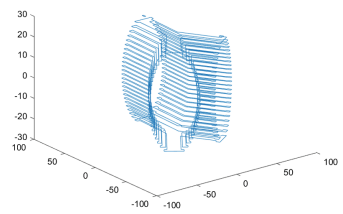

Fig. 15. Air duct of exp_BT for $(90,90)$ or $(0,90)$.

As expected, the algorithms found some solutions that have the same build orientation. It is the case of the $(180,180)$ or $(0,0)$ for the minimization of the volumetric error, $\exp _{-} V E$, and similar situations happen in the optimization of the support area and build time, experiments $\exp _{-} S A$ and $\exp _{-} B T$ (Fig. 15). As previously mentioned, the minimization of the total number of layers can reduce the part building time. Thus the optimal solutions of $\exp _{-} B T$ are consistent regarding the structure building direction.

Next we will present the figures related to build orientation problem of the Rocket Shot model. Note that the obtained figures related to experiment $\exp _{-} V E$ when the solution angles are $(0,0)$ and $(180,180)$ are the same (see Fig. 16) as well as for $(0,180)$ and $(180,0)$ (see Fig. 17). The solutions obtained by exp_BT are presented in Fig. 18.

Regarding the solution angles $(0,0)$ and $(180,180)$ seem to be the best direction (see Fig. 16). Since the solutions $(0,180)$ and $(180,0)$ have higher values of the objective function (see Table 1).

The build orientation directions produced by the global optimization algorithms for the 45 degree short model are depicted in Figs. 19, 20 and 21. The numerical results indicate that different angles $x$ and $y$ may result in the same direction of construction as had already occurred for the other models. 


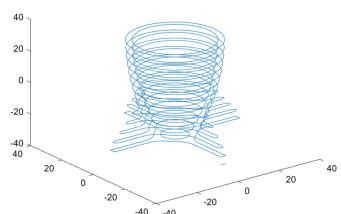

Fig. 16. Rocket shot of $\exp _{-} V E$ and $\exp _{-} S A$ for $(0,0)$ or $(180,180)$.

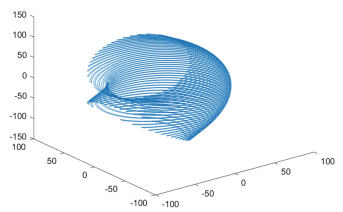

Fig. 19. 45 degree short of $\exp _{-} V E$ and $\exp _{-} S A$ for $(90,135)$.

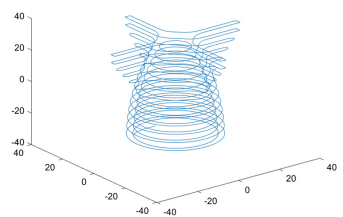

Fig. 17. Rocket shot of $\exp _{-} V E$ for $(0,180)$ and $(180,0)$.

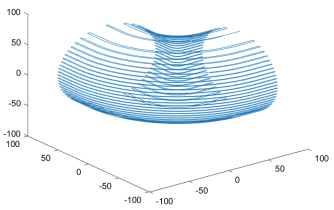

Fig. 20. 45 degree short of $\exp _{-} B T$ for $(180,0)$ or $(180,180)$.

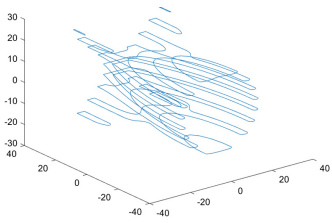

Fig. 18. Rocket shot of exp_BT for $(90,135)$ or $(90,45)$.

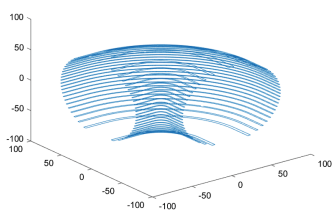

Fig. 21. 45 degree short of $\exp _{-} B T$ for $(0,0)$ or $(0,180)$.

\section{Conclusions and Future Work}

In this paper, it is presented a comparative study of two different global optimization methods, named the Electromagnetism-like and the Stretched Simulated Annealing algorithms, to solve the build orientation optimization problem. Numerical experiments have been carried out to analyse the behaviour of the EM and SSA algorithms when determining the best orientation to improve the surface accuracy of four 3D CAD models through three different measures, the volumetric error, the support area and the build time.

With this comparative study, we are able to conclude that both methods solve efficiently the optimization problem identifying different solutions in each model/measure. So, it is possible to conclude that for each presented model it is possible to build it with different orientations obtaining the same measure evaluation.

For future work we intend to solve the build orientation problem as a multiobjective problem and consider different and more complex models.

Acknowledgments. This work has been supported and developed under the FIBR3D project - Hybrid processes based on additive manufacturing of composites with long or short fibers reinforced thermoplastic matrix (POCI-01-0145-FEDER-016414), supported by the Lisbon Regional Operational Programme 2020, under the PORTUGAL 2020 Partnership Agreement, through the European Regional Development Fund (ERDF). This work was also supported by COMPETE: POCI-01-0145-FEDER- 
007043 and FCT - Fundação para a Ciência e Tecnologia within the Project Scope: UID/CEC/00319/2013.

\section{References}

1. Alexander, P., Allen, S., Dutta, D.: Part orientation and build cost determination in layered manufacturing. Comput. Aided Des. 30(5), 343-356 (1998)

2. Birbil, S.I., Fang, S.-C., Sheu, R.L.: On the convergence of a population-based global optimization algorithm. J. Global Optim. 30, 301-318 (2004)

3. Canellidis, V., Dedoussis, V., Mantzouratos, N., Sofianopoulou, S.: Pre-processing methodology for optimizing stereolithography apparatus build performance. Comput. Ind. 57(5), 424-36 (2006)

4. Canellidis, V., Giannatsis, J., Dedoussis, V.: Genetic-algorithm-based multiobjective optimization of the build orientation in stereolithography. Int. J. Adv. Manufact. Technol. 45, 714-730 (2009)

5. Gogate, A.S., Pande, S.S.: Intelligent layout planning for rapid prototyping. Int. J. Prod. Res. 46(20), 5607-5631 (2008)

6. Ingber, L.: Very fast simulated re-annealing. Math. Comput. Model. 12, 967-973 (1989)

7. Khana, I., Mateus, A., Lorgerc, K., Mitchella, G.R.: Part specific applications of additive manufacturing. Procedia Manufact. 12(2017), 89-95 (2017)

8. Lan, P., Chou, S., Chen, L., Gemmill, D.: Determining fabrication orientations for rapid prototyping with stereolithography apparatus. Comput. Aided Des. 29(1), 53-62 (1997)

9. Masood, S., Rattanawong, W., Iovenitti, P.: Part build orientations based on volumetric error in fused deposition modelling. Int. J. Adv. Manufact. Technol. 16, 162-168 (2000)

10. Parsopoulos, K., Plagianakos, V., Magoulas, G., Vrahatis, M.: Objective function stretching to alleviate convergence to local minima. Nonlinear Anal. 47, 3419-3424 (2001)

11. Pereira, A.I., Ferreira, O., Pinho, S.P., Fernandes, E.M.G.P.: Multilocal programming and applications. In: Zelinka, I., Snášel, V., Abraham, A. (eds.) Handbook of Optimization. Intelligent Systems Reference Library, vol. 38. Springer, Heidelberg (2013). https://doi.org/10.1007/978-3-642-30504-7_7

12. Phatak, A.M., Pande, S.S.: Optimum part orientation in rapid prototyping using genetic algorithm. J. Manuf. Syst. 31(4), 395-402 (2012)

13. Rocha, A.M.A.C., Silva, A., Rocha, J.G.: A new competitive implementation of the electromagnetism-like algorithm for global optimization. In: Gervasi, O., Murgante, B., Misra, S., Gavrilova, M.L., Rocha, A.M.A.C., Torre, C., Taniar, D., Apduhan, B.O. (eds.) ICCSA 2015. LNCS, vol. 9156, pp. 506-521. Springer, Cham (2015). https://doi.org/10.1007/978-3-319-21407-8_36

14. Thrimurthulu, K., Pandey, P.M., Reddy, N.V.: Optimum part deposition orientation in fused deposition modeling. Int. J. Mach. Tools Manuf 44(6), 585-94 (2004)

15. Villalpando, L., Eiliat, H., Urbanic, R.J.: An optimization approach for components built by fused deposition modeling with parametric internal structures. Procedia CIRP 17, 800-805 (2014)

16. Zhao J.: Determination of optimal build orientation based on satisfactory degree theory for RPT. In: Ninth International Conference on Computer Aided Design and Computer Graphics (CAD-CG 2005) (2005). p. 6 